\title{
Residual Effects Caused by a Past Mycovirus Infection in Fusarium circinatum
}

\author{
Cristina Zamora-Ballesteros ${ }^{1,2, *} \mathbb{C}$, Brenda D. Wingfield ${ }^{3}\left(\mathbb{D}\right.$, Michael J. Wingfield ${ }^{3}$, Jorge Martín-García ${ }^{1,2}$ \\ and Julio J. Diez ${ }^{1,2}$
}

1 Sustainable Forest Management Research Institute, University of Valladolid-INIA, 34004 Palencia, Spain; jorgemg@pvs.uva.es (J.M.-G.); juliojavier.diez@uva.es (J.J.D.)

2 Department of Vegetal Production and Forest Resources, University of Valladolid, 34004 Palencia, Spain

3 Department of Biochemistry, Genetics and Microbiology, Forestry and Agricultural Biotechnology Institute, University of Pretoria, Pretoria 0002, South Africa; brenda.wingfield@fabi.up.ac.za (B.D.W.); Mike.Wingfield@up.ac.za (M.J.W.)

* Correspondence: cristinazamoraballesteros@gmail.com

Citation: Zamora-Ballesteros, C.; Wingfield, B.D.; Wingfield, M.J.; Martín-García, J.; Diez, J.J. Residual Effects Caused by a Past Mycovirus Infection in Fusarium circinatum.

Forests 2021, 12,11. https:/ /dx.doi.org/ 10.3390/f12010011

Received: 8 October 2020

Accepted: 22 December 2020

Published: 24 December 2020

Publisher's Note: MDPI stays neutral with regard to jurisdictional claims in published maps and institutional affiliations.

Copyright: (c) 2020 by the authors. Licensee MDPI, Basel, Switzerland. This article is an open access article distributed under the terms and conditions of the Creative Commons Attribution (CC BY) license (https: / / creativecommons.org/ licenses/by/4.0/).

\begin{abstract}
Mycoviruses are known to be difficult to cure in fungi but their spontaneous loss occurs commonly. The unexpected disappearance of mycoviruses can be explained by diverse reasons, from methodological procedures to biological events such as posttranscriptional silencing machinery. The long-term effects of a virus infection on the host organism have been well studied in the case of human viruses; however, the possible residual effect on a fungus after the degradation of a mycovirus is unknown. For that, this study analyses a possible residual effect on the transcriptome of the pathogenic fungus Fusarium circinatum after the loss of the mitovirus FcMV1. The mycovirus that previously infected the fungal isolate was not recovered after a 4-year storage period. Only 14 genes were determined as differentially expressed and were related to cell cycle regulation and amino acid metabolism. The results showed a slight acceleration in the metabolism of the host that had lost the mycovirus by the upregulation of the genes involved in essential functions for fungal development. The analysis also revealed a weak expression in the annotated genes of the RNA silencing machinery. To our knowledge, this is the first time that a potential residual effect on the host transcriptome caused by the past infection of a mycovirus is reported.
\end{abstract}

Keywords: pine pitch canker disease; RNA-Seq; mitoviruses; transcriptome; differentially expressed genes

\section{Introduction}

Mycoviruses are ubiquitous in fungi [1], generally causing cryptic and persistent infections [2,3]. However, some of them are associated with variable effects on their hosts, from beneficial impacts [4,5] or mutualistic association [6] to deleterious effects [7-10]. These negative impacts usually reduce the physical integrity of the host fungus (hypovirulence). So far, the mycoviruses associated with this phenomenon have been reported in several plant pathogenic fungi, including Ophiostoma novo-ulmi, Botrytis spp., and Fusarium graminearum [11]. The model case of virocontrol of a forest disease is that of Cryphonectria parasitica hypovirus 1 (CHV1), the mycovirus of the highly destructive fungus of chestnut blight Cryphonectria parasitica. This hypovirus is used successfully as a biocontrol agent against chestnut blight in Europe and United States [12,13]. In a recent study, it has been demonstrated how a small DNA mycovirus of the necrotrophic pathogen Sclerotinia sclerotiorum is able to suppress the host pathogenicity and, in turn, stimulate the plant's growth and defences [14]. Interestingly, this mycovirus acts as a vaccine for plants.

Fungal viruses are thus good candidates for the biological control of plant diseases [15]. There is a growing body of literature focused on the study of fungal viruses with the aim of using virocontrol $[3,16,17]$. Three mycoviruses were recently reported from 
Fusarium circinatum, the causal agent of the disease commonly known as pine pitch canker (PPC) on pine trees $[18,19]$. The F. circinatum mycoviruses belong to the genus Mitovirus in the family Narnaviridae [18]. Species from this genus are capsidless ssRNA viruses that inhabit mitochondria and have genomes in the range of 2.3 to $3.5 \mathrm{~kb}[8,20]$.

To effectively study the effect of viruses on their host, virus-free and virus-infected isogenic lines are needed. The most common way of achieving this purpose is to remove the virus from infected strains. This can be done using different methods, such as the generation of monosporic isolates as well as antibiotic or thermal treatments [21]. It is also reported that unintentional loss of these mycoviruses can also occur during culturing and long-term storage of the fungi [22]. While loss of mycoviruses seem to be common, limited data exist regarding the stability of mycoviruses in their host.

A well-characterized fungi defence against virus infection from fungi is RNA silencing. The RNA-induced silencing complex (RISC) is an internal defence mechanism that protects genomes against invasion by mobile genetic elements such as viruses [23]. This machinery includes Dicer-like proteins that process viral structured dsRNA into small interfering RNAs (siRNAs), and Argonaute-like proteins that unwind the paired strands, degrade one of them, and use the other (aberrant RNA) as a template to identify similar sequences. The Argonaute-RISC complex then degrades the viral target, resulting in an antiviral response [24-26]. RNA silencing was first observed in C. parasitica where deletion of genes encoding Dicer- or Argonaute-like proteins could lead to severe growth defects in the strains infected by CHV1 but not the virus-free strains $[27,28]$. Furthermore, the presence of virus-derived siRNAs (vsiRNA) in C. parasitica, Magnaporthe oryzae [29], and Aspergillus nidulans [24] demonstrated the existence of active antiviral defence in filamentous fungi. Moreover, despite the fact that mitoviruses presumably replicate into the mitochondria, a proper functioning of RISC has been reported in members of the Mitovirus genus $[30,31]$. This possible biological explanation may lie behind the degradation of mycoviruses in their fungal hosts.

Loss of viruses that cause a symptomatic infection in the host essentially eliminates the symptoms. Nevertheless, little attention has been paid to the potential long-term functional impact of the loss of viruses on host organisms. In fact, to our knowledge, the potential residual effects result of a spontaneous virus loss event have only been reported on human viruses, such as the hepatitis B virus and West Nile virus [32], that trigger chronic diseases. More recent is the worrying clinical sequelae of severe acute respiratory syndrome coronavirus 2 (SARS-CoV-2) post-infection in the patients [33].

The original aim of this study was to compare two isogenic fungal lineages, one which contained the virus FcMV1 ( $\mathrm{Fc} 072 \mathrm{v})$ and one that had been cured of this virus ( $\mathrm{Fc} 072)$. However, during the study it was found that the isolate $\mathrm{Fc} 072 \mathrm{v}$ had in fact spontaneously lost this virus. This thus presented us with the opportunity to investigate whether there were differences in gene expression between two isogenic lineages, one that had very recently lost the virus FcMV1 and one that had been cultured for some time without this virus.

\section{Materials and Methods}

\subsection{Fungal Culture, Sample Preparation, RNA Extraction, and High-Throughput Sequencing}

The F. circinatum isolate $(\mathrm{Fc} 072 \mathrm{v})$ used in this work belongs to mating type 2 (MAT-2) and was isolated from an infected Pinus radiata tree located in the North of Spain (Cantabria, Spain). This isolate initially harboured the mycovirus "FcMV1". An isolate without this virus ( $\mathrm{Fc} 072)$ was obtained by monosporic culture in 2014 [19,34]. Likewise, after four years of storage, the unintentional loss of mycovirus "FcMV1" was confirmed according to Martínez-Álvarez et al. [18] (Table 1). 
Table 1. Presence (+) or loss/absence (-) of the mitovirus FcMV1 of Fusarium circinatum, detected by polymerase chain reaction (PCR).

\begin{tabular}{ccccc}
\hline \multirow{2}{*}{ Mycovirus } & \multicolumn{2}{c}{ Previous 4-Year Storage } & \multicolumn{2}{c}{ After 4-Year Storage } \\
\cline { 2 - 5 } & Fc072 & Fc072v & Fc072 & Fc072v \\
\hline $\begin{array}{c}\text { Fusarium circinatum mitovirus 1 } \\
\text { (FcMV1) }\end{array}$ & - & + & - & - \\
\hline
\end{tabular}

Each F. circinatum isolate ( $\mathrm{Fc} 072$ and $\mathrm{Fc} 072 \mathrm{v})$ was cultured in three Petri dishes containing PDA medium (3.90\% w/v potato dextrose agar, Scharlab S.L., Barcelona, Spain). After culturing for a week in darkness at $25^{\circ} \mathrm{C}$, each colony was subcultured in three Erlenmeyer flasks with $100 \mathrm{~mL}$ of PDB medium $(2.40 \% \mathrm{w} / \mathrm{v}$ potato dextrose broth, Scharlab S.L., Barcelona, Spain) and incubated in an orbital shaker at 150 cycles for 4 days at $25^{\circ} \mathrm{C}$. The filtered tissue was flash frozen in liquid nitrogen and ground to a fine powder using a mortar and pestle. The powder of the three flasks were pooled before RNA extraction, which was performed using the Spectrum ${ }^{\mathrm{TM}}$ Plant Total RNA Kit (Sigma Aldrich, St. Louis, $\mathrm{MO}$, USA) following the manufacturer's protocols. Any contaminating traces of genomic DNA from RNA were removed by on-column DNase Digestion (DNASE10-1SET, SigmaAldrich, St. Louis, MO, USA). The concentration and purity of the RNA was measured by estimating the absorbance ratios $\mathrm{A}_{260} / \mathrm{A}_{230}$ and $\mathrm{A}_{260} / \mathrm{A}_{280}$ (NanoDrop 2000 Spectrophotometer, Thermo Fisher Scientific, Waltham, MA, USA). RNA integrity was checked by electrophoresis on an agarose gel (1\% TAE).

The RNA was sent to Fasteris SA (Switzerland; https:/ / www.fasteris.com) for Illumina Stranded RNA library construction and HTS (Illumina HiSeq 4000, Illumina Inc., San Diego, CA, USA). The sample processing consisted of purification using poly(A) selection, chemical fragmentation, synthesis of single- and double-stranded cDNA using random hexamer priming, end repair, ligation of the $3^{\prime}$ and $5^{\prime}$ adapters, and amplification by PCR. The 75-bp raw paired-end reads were generated by the Illumina HiSeq 4000 platform across only one Illumina lane, in order to avoid technical errors due to sample position in the sequencer.

\subsection{RNA-Seq Analysis}

The software package CLC Genomics Workbench 6.0.1 (CLC bio, Aarhus, Denmark) was used to discard poor-quality terminal nucleotides. In particular, two nucleotides were removed from the $5^{\prime}$ terminal of the reads. This software was used to align the trimmed sequence reads to the annotated F. circinatum reference genome. To ensure optimal alignment, we used a filtering threshold of 0.5 for the length fraction and a similarity parameter value of 0.8 . Thus, at least $50 \%$ of an individual read needed to match the reference sequence at a similarity of $>80 \%$ for the read to be used in the mapping. Comparisons of the expression levels between the samples were done according to Mortazavi et al. [35]. Data normalization of each sample was carried out by dividing the number of reads mapping to each gene by its length and by the total number of reads sequenced across the transcriptome (reads per kilobase per million mapped reads, or RPKM). A quantile normalization algorithm was also applied on the RPKM values to account for technical variability in the RNA-Seq data [36].

Baggerley's $Z$ test on the expression proportions was performed to test the RPKM differences between the samples [37], using the normalized RPKM values. This test compares the proportions of counts in a group of samples against those of another group of samples (i.e., Fc072 vs. Fc072v), where the samples are given different weights depending on their sizes (total counts). The weights are obtained by assuming a $\beta$ distribution on the proportions in each group, and estimating these, along with the proportion of a binomial distribution, by the method of moments. The result is a weighted t-type test statistic. The Benjamini-Hochberg false discovery rate (FDR) method was used for multiple test correction [38]. In this study, a conservative threshold for identifying differentially 
expressed (DE) genes was used, ignoring those genes showing lower effects. DE genes were considered only if they displayed a 2-fold change in expression at an FDR-corrected $p$-value of 0.05 .

In an attempt to identify viral expression, F. circinatum mitoviruses genomes from the NCBI Taxonomy Database (https:/ / www.ncbi.nlm.nih.gov/taxonomy) were used as queries in the local mapping tool against the libraries in CLC Genomics Workbench. The mapping approach was the same as that described above (length fraction $=0.5$; similarity parameter $=0.8$ ).

Genes known to be involved in post-transcriptional gene silencing in F. graminearum were investigated for their presence and possible over or under-regulated expression in the two conditions. These included the RNA silencing core components already known to be present in F. graminearum: two Argonaute-like, two Dicer-like, and five RNA-directed RNA polymerase genes [25]. Also included in this list were an exonuclease QIP (QDE2interacting protein) that is involved in the activation of RISC and a QDE-3 (RecQ DNA helicase homologous to the Werner/Bloom Syndrome proteins) required for recognizing aberrant RNA [39]. In addition, an MRPL3 (RNase III domain-containing protein) identified in Neurospora crassa that could collaborate with Dicer [40] was included (Table S8). The gene sequences were downloaded from the NCBI Gene Database (www.ncbi.nlm.nih.gov/gene) and identified by blasting them against the draft genome assemblies of F. circinatum using local BLAST.

\subsection{Gene Ontology (GO) Enrichment Analysis}

To shed light on the functional processes that may have been modified by a past infection of mycovirus FcMV1, enrichment of the differentially expressed genes in gene ontology (GO) terms was carried out. Because of the poor annotation for F. circinatum genes, all genes (a total of 14,865) were annotated using the BLAST2GO program [41]. BLAST2GO was also used to predict the protein domains through InterProScan 5 [42] as well as to perform Gene Ontology (GO) and Enzyme Code (EC) mapping.

GO enrichment analyses were performed using Fisher's exact test (FET) in the BLAST2GO program to determine whether any GO terms were enriched in the DE gene list with respect to all of the GO terms of the complete F. circinatum transcriptome [41]. It was run with the default settings, taking a false discovery rate (FDR) $<0.05$. In addition, the highly expressed genes were also subjected to FET testing for GO term enrichment, with a $p$-value of 0.05 or less indicating significant enrichment. The identified GO terms were classified into three ontologies: biological process, cellular component, and molecular function. General GO terms (parent terms) were removed to keep the most specific terms using the REViGO 2017 [43] tool with the "small, 0.5 allowed similarity" setting. The REViGO output was then visualized using Cytoscape v3.8.0 (ISB, Seattle, WA, USA).

\section{Results}

\subsection{RNA-Seq Statistics}

High-quality RNA (RIN > 7) was extracted from each sample used in this study. Illumina HiSeq sequencing of the F. circinatum libraries produced from 54,741,864 to $72,397,413$ reads with a length of 75 nucleotides (Table 2). The raw sequencing data have been deposited in the National Center for Biotechnology Information (NCBI) Sequence Read Archive (SRA) database under the following accession number: PRJNA666978. After quality trimming (two nucleotides per read) the Phred-scale quality score was higher than 40 in at least $75 \%$ of the sequences. The mapping approach allowed an efficient alignment of the reads generated for the respective samples against the F. circinatum reference genome, with $98 \%$ of fungal reads (Table 2). Unmapped reads were most likely the result of sequencing errors, reads from repetitive or unassembled genome regions [44], and the presence of viral sequences. 
Table 2. Summary of the sequence reads.

\begin{tabular}{ccc}
\hline Samples & Number of Raw Reads $^{\text {a }}$ & Number of Mapped Fungal Reads $^{\mathbf{b}}$ \\
\hline (1) Fc072 & $58,988,010$ & $57,818,686(98.02 \%)$ \\
\hline (2) Fc072 & $59,120,902$ & $57,957,023(98.03 \%)$ \\
\hline (3) Fc072 & $66,538,581$ & $65,199,515(97.99 \%)$ \\
\hline (4) Fc072V & $54,741,864$ & $53,618,877(97.95 \%)$ \\
\hline (5) Fc072V & $57,606,375$ & $56,514,903(98.11 \%)$ \\
\hline (6) Fc072V & $72,397,413$ & $70,933,962(97.98 \%)$ \\
\hline
\end{tabular}

${ }^{a}$ Passed filter clusters that fulfil the default Illumina quality criteria; ${ }^{b}$ Reads mapped to the

F. circinatum reference genome in pairs and in broken pairs.

\subsection{Detection of Gene Expression}

Out of the 14,865 predicted genes in the F. circinatum genome ( $44 \mathrm{Mb})$, approximately $77 \%$ of the genes were expressed similarly across all the libraries in this study. A transcript was considered to be expressed if at least 3 unique gene reads were mapped to its annotation and if it was detected above a threshold of 0.1 normalized RPKM value [45]. The number of highly expressed genes (RPKM > 1000) was similar in both conditions (Table 3). Unfortunately, $50 \%$ of these genes were not annotated or encoded hypothetical proteins with conserved domains. The genes that could be assigned to a probable function were enriched for processes such as regulation of gene expression, protein binding, and response to stimulus (Table S3). In particular, the GO analysis identified many items associated with the transcriptional and translational machinery. RNA polymerase II transcription preinitiation complex assembly (GO:0045899), activation of transcription by nitrogen catabolites (GO:0001080), rRNA transcription (GO:0009303), nucleosome assembly (GO:0006334), and cytoplasmatic translational elongation (GO:0002182) were enriched.

Table 3. RNA-Seq gene expression results for the two conditions.

\begin{tabular}{ccc}
\hline Category & Fc072 & Fc072V \\
\hline Highly expressed genes $(>1000$ RPKM) & 36 & 34 \\
\hline Medium expressed genes $(\geq 10$ to $1000 \mathrm{RPKM})$ & 7172 & 7158 \\
\hline Lowly expressed genes $(<10 \mathrm{RPKM})$ & 4290 & 4292 \\
\hline RPKM values $\geq 0.2$ & 11,494 & 11,484 \\
\hline
\end{tabular}

In the isolate previously infected by the mitovirus $(\mathrm{Fc} 072 \mathrm{v}), 34$ genes were highly expressed with only three being unique to this condition (Table S4). Canonical glycolysis (GO:0061621), gluconeogenesis (GO:0006094), and pyruvate biosynthetic process (GO:0042866) were enriched GO terms. All three of these GO terms are involved in carbohydrate metabolism. Transcripts involved in the metabolic process of reactive oxygen species (ROS) (GO:0072593) and osmotic stress (GO:0071470) were upregulated. A set of $\mathrm{GO}$ terms associated with the oxidative stress response, such as detection of oxidative stress (GO:0070994), cellular response to oxidative stress (GO:0034599), and apoptotic process (GO:0006915), were enriched. In addition, positive regulation of the stress-activated MAPK cascade (GO:0032874) and Mcs4 RR-MAPKKK complex (GO:1990315), which play a pivotal role in the regulation of stress responses, were over-represented. Nucleotide catabolic process (GO:0009166) and purine ribonucleoside monophosphate biosynthetic process (GO:0009168), both involved in the synthesis and degradation of nucleotides, were also over-represented. Other terms related to the regulation of all these processes, including heme transport (GO:0015886), GAPDH activity (GO:0004365), NAD biosynthetic process (GO:0009435), and ATP biosynthesis process (GO:0006754), were upregulated as well (Table S5). 
Out of the genes expressed in the $\mathrm{Fc} 072$ isolate, 36 genes were highly expressed, with five unique to this condition. The only gene that could be assigned to the probable function was phosphatidylserine decarboxylase proenzyme 2 (Table S6).

The results of the local mapping using the genomes of the mitoviruses of $F$. circinatum confirmed the absence of FCMV1 in both treatments, as checked by PCR. No reads of any of the libraries analysed in this study matched the FcMV1 genome. Surprisingly, a total of 4100 reads from the six libraries were mapped against the FcMv2-1 genome, confirming the hitherto unknown presence of this mitovirus in the isolates in this study (Table 4).

Table 4. Mapping summary statistics, with the reference genomes of mitoviruses FcMV1 and FcMV21 as queries.

\begin{tabular}{cccccccc}
\hline \multirow{2}{*}{$\begin{array}{c}\text { Mycovirus } \\
\text { Genome }\end{array}$} & $\begin{array}{c}\text { Reference } \\
\text { Length }\end{array}$ & \multicolumn{7}{c}{ Reads Mapped to Genome * $^{*}$ (1) Fc072 } & (2) Fc072 & (3) Fc072 & (4) Fc072v & (5) Fc072v & (6) Fc072v \\
\hline FcMV1 & 2419 & 0 & 0 & 0 & 0 & 0 & 0 \\
\hline FcMV2-1 & 2193 & 575 & 452 & 805 & 835 & 737 & 696 \\
\hline \multicolumn{7}{c}{ * Reads mapped in pairs and in broken pairs. }
\end{tabular}

\subsection{Expression of Genes Involved in Post-Transcriptional Gene Silencing}

Based on similarity, genes involved in post-transcriptional gene silencing were identified and their expression was examined. Using the F. graminearum and N. crassa genes as queries, we identified the homologous loci from the F. circinatum genome (Table S8). The gene that encodes the Dicer protein DCL-1, which plays presumably an antiviral role in F. graminearum, and one of the RNA-dependent RNA polymerases, were not found. None of the genes identified showed evidence of differential expression (Table S9) and were categorized as medium/lowly expressed genes in the study.

\subsection{Differentially Expressed Genes}

Out of the expressed genes, only 14 were differentially expressed (DE; Figure S1), as they displayed a 2.0-fold change at an FDR of $\mathrm{q} \leq 0.05$ during pairwise comparisons between the two treatments (with residual effects and without virus). A probable function of 12 out of the 14 transcripts could be assigned (Table 5, Figure S2). All the DE genes were upregulated, except a cytochrome P450 that encodes an oxidative enzyme required in lovastatin biosynthesis (Figure S3). To gain insights into the functions of the differentially expressed genes, as a residual effect of the FcMV1 infection, we analysed them for enriched GO terms; all listed in Table S7. The genes indicated significant enrichment with a $p$-value of 0.05 or less for biological regulation, transport, metabolic, and cellular processes (Figures S4 and S5). The majority of upregulated functional groups were related to cellular component organization and cell cycle regulation, such as positive regulation of formin-nucleated actin cable assembly (GO:0090338), regulation of barbed-end actin filament capping (GO:2000812), mitotic actomyosin contractile ring assembly (GO:1903475), actin cortical patch organization (GO:0044396), actin monomer binding (GO:0003785), and cell cortex of cell tip (GO:0051285). In addition, terms involved in the biosynthesis of the methionine pathway, including homoserine metabolic process (GO:0009092) and methionine biosynthetic process (GO:0009086), were enriched. The NAD, NADH, and NADP metabolic processes (GO:0019674, GO:0006734, and GO:0006739) that modulate numerous biological events were enriched as well. Transcripts involved in the macromolecule metabolic process (GO:0043170) were under-represented in this analysis. More results of Blast2GO are represented in Figures S6 and S7. 
Table 5. GO terms of the differentially expressed genes. $\uparrow=$ upregulated by the FcMV1 residual effect; $\downarrow=$ downregulated by the FcMV1 residual effect.

\begin{tabular}{lccc}
\hline Gene Locus & Gene Function & $\begin{array}{c}\text { Fold } \\
\text { Change }\end{array}$ & $\begin{array}{c}\text { FDR } p \text {-Value } \\
\text { Correction }\end{array}$ \\
\hline FCIRG_04875 & Nicotianamine synthase 3 & $2.22 \uparrow$ & $8.29 \times 10^{-5}$ \\
\hline FCIRG_04876 & O-acetylhomoserine (thiol)-lyase & $2.62 \uparrow$ & 0 \\
\hline FCIRG_04877 & Carnosine synthase 1 & $2.22 \uparrow$ & 0.02 \\
\hline FCIRG_04879 & Uncharacterized protein & $2.01 \uparrow$ & 0 \\
\hline FCIRG_04880 & GA4 desaturase & $2.43 \uparrow$ & 0 \\
\hline FCIRG_04881 & Probable O-acetylhomoserine (thiol)-lyase & $2.53 \uparrow$ & $9.13 \times 10^{-9}$ \\
\hline FCIRG_04882 & Related to oxidoreductase & $2.24 \uparrow$ & 0 \\
\hline FCIRG_04883 & Related to naringenin, 2-oxoglutarate & $2.33 \uparrow$ & 0 \\
\hline FCIRG_04972 & 3-dioxygenase & $2.34 \uparrow$ & 0.01 \\
\hline FCIRG_05571 & Profilin & $2.06 \uparrow$ & $8.29 \times 10^{-5}$ \\
\hline FCIRG_07760 & Related to human TGR-CL10C & $2.19 \uparrow$ & 0 \\
\hline FCIRG_11031 & ABC transporter YOR1 & $2.18 \uparrow$ & 0.02 \\
\hline FCIRG_14348 & No significant similarity & $2.19 \uparrow$ & 0 \\
\hline FCIRG_14896 & Probable CYP450 monooxygenase (lovA) & $3.20 \downarrow$ & 0 \\
\hline
\end{tabular}

\section{Discussion}

Although the spontaneous loss of mycoviruses after a storage period is well known by mycovirus researchers, as far as we are aware this is the first time that a potential residual effect on the host transcriptome caused by a past infection of a mycovirus is reported. This study represents a preliminary attempt to analyse a genome-wide transcriptome by RNASeq of the pathogenic fungus F. circinatum infected by F. circinatum mitovirus 1 (FcMV1) and its subsequent spontaneous loss.

The transcriptomic analysis confirmed the loss of the FcMV1 mycovirus, since no reads of the set of the sequenced libraries matched the FcMV1 genome. Unfortunately, the disappearance of this mycovirus in the $\mathrm{Fc} 072$ strain made it impossible to include this strain harbouring FcMV1 in the transcriptomic analysis. An unexpected result emerging from the data was the detection of FcMV2-1 in all the libraries. This virus was not detected before by using the conventional method of PCR and its specific primers [19]. Similar findings also occurred in a study with Heterobasidion annosum, where cryptic mitovirus infections were not detected by dsRNA extraction but by RNA deep sequencing and RNA-Seq analysis [31,46]. The fact that mycoviruses can be detected or not depends on the state of replication, the titre, and the sample handling. The use of conventional PCR in viral detection is a widely used method and considered as highly sensitive [47]. However, if the number of copies of the target molecule is very scarce, the use of realtime PCR (qPCR) or High-Throughput Sequencing (HTS) technologies are more sensitive and, therefore, recommended [48-50]. The results regarding the viral accumulation of FcMV2-1 (0.001\%) are consistent with previous transcriptome analysis where the amount of virus accumulation was very low [51]. A low rate of viral replication (latency) at the time when the RNA was extracted could be an explanation. As viral replication relies on the host translational machinery to produce viral proteins, an increment in the expression of translational genes is expected. Indeed, the translational machinery was highly expressed in all the samples; however, it cannot be claimed that FcMV2-1 was using this machinery for its replication. Another biological explanation behind the unsuccessful detection of FcMV21 by conventional PCR could be a high activity of the RISC machinery in the host, the protection mechanism of eukaryotes from viral infections. Nevertheless, the examination 
of the expression of the posttranscriptional gene silencing showed a low activity of the RISC core in all the samples. Therefore, the factors that regulate the FcMV2-1 titre must be further studied.

The presence of the cryptic mycovirus FcMV2-1 was reflected in the reads mapped to its genome. The results of the mapping showed a similar number of FcMV2-1 reads in both conditions (Welch's two-sample $\mathrm{t}$-test; $\mathrm{t}=-1.305, p=0.29$ ). It is reasonable to assume that the viral abundance in each individual library is correlated with the relative abundance of the virus in the host [52]. Thus, it is likely that both isolates had the same infection load of FcMV2-1 and that FcMV2-1 would be affecting the transcriptome of its host in an equal manner and probably not contributing to the differential gene expression.

In the present study, the RNA-Seq-based genome-wide expression analysis showed that approximately $77 \%$ of genes of $F$. circinatum were expressed. The residual effect caused by a past FcMV1 infection was only represented by 12 known DE genes. Although the value concurs with the number of $\mathrm{DE}$ genes reported in response to the coinfection of four mycoviruses (FgV1, FgMV2, FgMV3, and FgMV4) in F. graminearum [53], other studies found a much larger number of DE genes as a result of mycovirus infections. For example, a total of 683 and 848 genes were DE in H. annosum and Phytophthora infectans by the infection of the partitivirus HetPV13-an1 and PiRV-2, respectively [46,54]. Likewise, F. graminearum hypovirus 1 (FgHV1) caused a significant alteration in a total of 378 genes [55]. This could suggest that the residual effect of a past mycovirus infection is limited to a few genes and functions in comparison with those affected in the presence of the mycovirus.

In particular, out of the $12 \mathrm{DE}$ genes, only "cytochrome P450 monooxigenase (lov $A)^{\text {" }}$ was downregulated by the FcMV1 post-infection. This cytochrome encodes an oxidative enzyme essential for the production of lovastatin biosynthesis [56], a fungal secondary metabolite. The gene $\operatorname{lov} A$ is considered as a gene required by the pathogenic fungus to establish infections [57]. In addition, this gene product may also be involved in generating compounds that exercise control over expression of the $\beta$-oxidation system [58], which are necessary for conidial germination and invasive growth of the host [59].

Actin filaments are one of the major components that constitute the cytoskeleton of the eukaryotic cells. These fibres are directly involved in morphogenesis, cytokinesis, and organelle transport in filamentous fungi [60]. Because actin is essential for determining the shape of the cell, it takes on great importance during mitosis. A large number of ontology terms involved in the actin cytoskeleton were over-represented by the upregulated DE genes. Some of them were involved in the organization and formation of structures that serve as tracks for intracellular transport in the process of cytokinesis. These terms include actin cortical patches, formin-nucleated actin cables, and mitotic actomyosin contractile rings. Assembly and constriction of the actomyosin ring occur during cell division in fungal cells [61]. This suggests that the fungal cultures that had lost the virus have a higher cellular development than the virus-free isolate.

Some of the upregulated genes, considered as the residual effect of a past infection, encode proteins involved in the response and regulation of oxidative stress. The ATPbinding cassette transporter YOR1, a drug efflux pump, also intervenes in pathogenicity and stress response [62]. A gene related to naringenin 2-oxoglutarate 3-dioxygenase also has been upregulated by FcMV1 post-infection; these gene products are involved in the biosynthesis of flavonoids, recently described in the fungi kingdom [63]. Although the production of this secondary metabolite has an important role in plant defence, it is unclear what role this plays in fungi. Due to the antioxidant effects of flavonoids, we could think they can be important in fungal counter-attack against reactive oxygen species (ROS). The ROS metabolic process was present among the items identified in the GO term enriched analyses of the highly expressed genes, uniquely in the cultures that had lost the mycovirus. Together with ROS, multiple terms enriched were involved in redox regulation and response to osmotic stress, suggesting a counterbalance and defence reaction of $F$. circinatum triggered by an own oxidative burst. Other researchers found a cellular response to oxidative stress under mycovirus infection [46,55]. 
Osmotic stress or high levels of ROS cause stress in the fungus, resulting in growth cessation. ROS are highly potent oxidants and their inappropriate regulation causes damage of macromolecules such as DNA and proteins, which could lead to an apoptoticlike cell death. However, local bursts of ROS regulate different cellular functions, such as cell proliferation, cell differentiation, and ion transport [64]. The thin line between a destructive and signalling role of ROS in the cell depends on the equilibrium between their production and scavenging [65]. An increment in intracellular ROS concentration before each stage of the development cycle has been demonstrated in different fungi [66]. Taking all together, these results seem to suggest that the oxidative burst could not be related to a defence mechanism against a viral infection, but to the development of the colony, inducing hyphae and macroconidium formation. This would be consistent with a preliminary study of FcMV1 infection, where the mitovirus caused a slight increase in F. circinatum virulence [34]. Thus, the residual effect of a recent infection by FcMV1 could have somehow accelerated the metabolism of the F. circinatum cultures.

Interestingly, four genes in the upregulated group are implicated direct or indirectly in the methionine pathway. The methionine biosynthetic process and the homoserine metabolic process were also enriched upon FcMV1 post-infection. Allen et al. [67] found that strains of $C$. parasitica infected by the hypovirus $\mathrm{CHV} 1$ show transcript accumulation for genes involved in the methionine pathway. Similar results were also observed in a study of yeast-totivirus interaction, where a fungal response to the viral infection was suggested [68]. Due to its central role in metabolism, it could be predicted that the increment in the expression of genes involved in the methionine pathway would be affecting many metabolic and physiologic processes, such as protein synthesis and membrane integrity [67]. These alterations may constitute the consequences of the previous presence of FcMV1 on the stability of the F. circinatum genome. However, further experimental evidence must support this hypothesis.

RNA silencing is an evolutionarily conserved system in eukaryotic organisms. Besides its developmental regulation function in the organism, it has been demonstrated that RNA silencing also acts as an antiviral defence mechanism in filamentous fungi [69]. There was some evidence of the molecular antiviral response in F. circinatum. In a study of vsiRNA fragments using HTS, more than $50 \%$ of reads from each $F$. circinatum mitovirus strain ranged between 20 and $26 \mathrm{nt}$, the highest reported vsiRNA percentage in filamentous fungi [70]. In the present study, the expression of genes involved in gene silencing pathways was detected but no significative differences between the two isolates were observed. The presence of the mitovirus FcMV2-1 in all the cultures did not trigger a strong response in the post-transcriptional silencing machinery. The levels of expression of these genes were low with an average of 7 to 8 RPKM. A slightly higher expression of silencing genes was observed in H. annosum, with 49-50 RPKM [46], which may be explained by the fact that the cultures were infected by two identified mitoviruses. The results reported here are consistent with a recent study where it has been demonstrated that $C$. parasitica mitovirus 1 is unaffected by the RNA silencing mechanism [71]. The possibility that the mitoviruses could escape the cytoplasmic RISC defences because of their subcellular localization still has to be addressed.

To our knowledge, this is the first time that a residual effect on the gene expression of a fungus caused by a past infection of a putative mycovirus has been reported. The limited number of genes differentially expressed reveals a low interference in the transcriptome on the host. However, the number of altered genes is not the only factor, the functions of the genes affected is also important. The present study shows that several essential functions were presumably upregulated by a recent infection of the putative mycovirus FcMV1. However, the present work represents a preliminary study based on genome-wide transcriptome and further experimental essays are needed to validate the residual effects caused by the mycoviruses. These results highlight the importance of understanding more about the viruses hosted by fungi isolates and their spontaneous losses. This study lays the 
groundwork for further studies on the loss of mycoviruses and their residual effects on the host.

\section{Conclusions}

The spontaneous loss of mycoviruses is common and it can lead to a long-term functional impact on the fungal hosts. RNA-Seq technology has allowed the analysis of the residual effect of the past infection of the mitovirus FcMV1 on the fungal pathogen Fusarium circinatum based on a genome-wide transcriptome. We demonstrated that the fungal isolate that had lost the mycovirus was experiencing a slight acceleration in metabolism, possibly due to the positive regulation of the genes involved in the essential functions for the development of the fungus.

Supplementary Materials: The following are available online at https:/ /www.mdpi.com/1999-490 7/12/1/11/s1, Figure S1: Venn diagram of the differentially expressed genes by the post-infection of the virus FcMV1. Figure S2: Sequences of the differentially expressed genes by the post-infection of the virus FcMV1 without a known function. Figure S3: Scatter plot representing the differentially expressed genes of this analysis. Xaxis: normalized means in condition free of FcMV1 infection. Y-axis: normalized means in condition of FcMV1 post-infection. Red dot: downregulated genes; green dot: upregulated genes. Figure S4: FET enriched GO terms associated with the differentially expressed genes of Fusarium circinatum previously infected by FcMV1. Figure S5: Distribution of GO terms represented in this analysis by biological processes, cellular components and molecular functions. Figure S6: Distribution of the sequences of the analysis in the InterProScan domains. Figure S7: Blast2GO blast results of the highest similarity species with higher Max ident and lower evalues. Table S1: RNA sequencing and genome mapping statistics. Table S2: Genes highly expressed of F. circinatum shared in both conditions. Table S3: FET enriched GO terms associated with the genes highly expressed of F. circinatum shared in both conditions. Table S4: Genes highly expressed in Fc072v cultures of F. circinatum. Table S5: FET enriched GO terms associated with the genes highly expressed in Fc072v cultures of F. circinatum. Table S6: Genes highly expressed in Fc072 cultures of F. circinatum. Table S7: FET enriched GO terms associated with the genes upregulated in F. circinatum by the FcMV1 residual effect. Table S8: Blast homology result of the genes implicated in post transcriptional gene silencing in Fusarium circinatum. Table S9: Expression ratios (RPKM and fold-change relative to the virus-free monosporic) of Fusarium circinatum genes involved in post transcriptional gene silencing.

Author Contributions: B.D.W., M.J.W. and J.J.D. conceived and designed the experiment; C.Z.-B. performed the experiments and analyzed the data; C.Z.-B. and J.M.-G. wrote the paper; B.D.W., M.J.W. and J.J.D. reviewed and editing the paper; J.J.D. supervised the project. All authors have read and agreed to the published version of the manuscript.

Funding: This article is based upon the work of COST Action FP1406 PINESTRENGTH (Pine Pitch Canker strategies for management of Gibberella circinata in greenhouses and forests) supported by COST (European Cooperation in Science and Technology) and project AGL2015-69370-R funded by MINECO (Spain). This study was made possible through the Interreg SUDOE project PLURIFOR "Transnational Plans for the Management of Forest Risks", and with the support of the Government of Cantabria (Spain). Research was supported by project PID2019-110459RB-I00 funded by MICINN (Spain) and FEDER (EU) budget as well as the project VA208P20 funded by JCYL (Spain).

Data Availability Statement: The datasets analyzed in this study can be found here: NCBI SRA with accession number: PRJNA666978.

Acknowledgments: We thank Jordán Muñoz-Adalia, Pablo Martínez-Álvarez, Diana Bezos and Carmen Romeralo for laboratory support. We also thank Lieschen de Vos for bioinformatics assistance.

Conflicts of Interest: The authors declare no conflict of interest.

\section{References}

1. Ghabrial, S.A.; Castón, J.R.; Jiang, D.; Nibert, M.L.; Suzuki, N. 50-Plus Years of Fungal Viruses. Virology 2015, 479-480, 356-368. [CrossRef] [PubMed]

2. Ghabrial, S.A.; Suzuki, N. Viruses of Plant Pathogenic Fungi. Annu. Rev. Phytopathol. 2009, 47, 353-384. [CrossRef] [PubMed] 
3. Pearson, M.N.; Beever, R.E.; Boine, B.; Arthur, K. Mycoviruses of filamentous fungi and their relevance to plant pathology. Mol. Plant Pathol. 2009, 10, 115-128. [CrossRef] [PubMed]

4. Hyder, R.; Pennanen, T.; Hamberg, L.; Vainio, E.J.; Piri, T.; Hantula, J. Two viruses of Heterobasidion confer beneficial, cryptic or detrimental effects to their hosts in different situations. Fungal Ecol. 2013, 6, 387-396. [CrossRef]

5. Ahn, I.-P.; Lee, Y.-H. A Viral Double-Stranded RNA Up Regulates the Fungal Virulence of Nectria radicicola. Mol. Plant-Microbe Interact. 2001, 14, 496-507. [CrossRef] [PubMed]

6. $\quad$ Márquez, L.M.; Redman, R.S.; Rodriguez, R.J.; Roossinck, M.J. Thermotolerance Generated by Plant/Fungal Symbiosis. Science 2007, 298, 1581. [CrossRef]

7. Anagnostakis, S.L.; Chen, B.; Geletka, L.M.; Nuss, D.L. Hypovirus Transmission to Ascospore Progeny by Field-Released Transgenic Hypovirulent Strains of Cryphonectria parasitica. Phytopathology 1998, 88, 598-604. [CrossRef] [PubMed]

8. Hong, Y.; Dover, S.L.; Cole, T.E.; Brasier, C.M.; Buck, K.W. Multiple mitochondrial viruses in an isolate of the Dutch elm disease fungus Ophiostoma novo-ulmi. Virology 1999, 258, 118-127. [CrossRef]

9. Xie, J.; Wei, D.; Jiang, D.; Fu, Y.; Li, G.; Ghabrial, S.; Peng, Y. Characterization of debilitation-associated mycovirus infecting the plant-pathogenic fungus Sclerotina sclerotorium. J. Gen. Virol. 2006, 87, 241-249. [CrossRef]

10. Castro, M.; Kramer, K.; Valdivia, L.; Ortiz, S.; Castillo, A. A double-stranded RNA mycovirus confers hypovirulence-associated traits to Botrytis cinerea. FEMS Microbiol. Lett. 2003, 228, 87-91. [CrossRef]

11. Xie, J.; Jiang, D. New Insights into Mycoviruses and Exploration for the Biological Control of Crop Fungal Diseases. Annu. Rev. Phytopathol. 2014, 52, 45-68. [CrossRef] [PubMed]

12. Rigling, D.; Prospero, S. Cryphonectria parasitica, the causal agent of chestnut blight: Invasion history, population biology and disease control. Mol. Plant Pathol. 2018, 19, 7-20. [CrossRef] [PubMed]

13. Double, M.L.; Jarosz, A.M.; Fulbright, D.W.; Davelos Baines, A.; Macdonald, W.L. Evaluation of Two Decades of Cryphonectria parasitica Hypovirus Introduction in an American Chestnut Stand in Wisconsin. Phytophatology 2018, 108, 702-710. [CrossRef] [PubMed]

14. Zhang, H.; Xie, J.; Fu, Y.; Cheng, J.; Qu, Z.; Zhao, Z.; Cheng, S.; Chen, T.; Li, B.; Wang, Q.; et al. A 2-kb Mycovirus Converts a Pathogenic Fungus into a Beneficial Endophyte for Brassica Protection and Yield Enhancement. Mol. Plant 2020, 13, 1420-1433. [CrossRef] [PubMed]

15. Hillman, B.I.; Annisa, A.; Suzuki, N. Viruses of Plant-Interacting Fungi. In Advances in Virus Research; Academic Press Inc.: New York, NY, USA, 2018; Volume 100, pp. 99-116; ISBN 9780128152010.

16. Muñoz-Adalia, E.J.; Fernández, M.M.; Diez, J.J. The use of mycoviruses in the control of forest diseases. Biocontrol Sci. Technol. 2016, 26, 577-604. [CrossRef]

17. García-Pedrajas, M.D.; Cañizares, M.C.; Sarmiento-Villamil, J.L.; Jacquat, A.G.; Dambolena, J.S. Mycoviruses in biological control: From basic research to field implementation. Phytopathology 2019, 109, 1828-1839. [CrossRef]

18. Martínez-Álvarez, P.; Vainio, E.J.; Botella, L.; Hantula, J.; Diez, J.J. Three mitovirus strains infecting a single isolate of Fusarium circinatum are the first putative members of the family Narnaviridae detected in a fungus of the genus Fusarium. Arch. Virol. 2014, 159, 2153-2155. [CrossRef]

19. Vainio, E.J.; Martinez-Alvarez, P.; Bezos, D.; Hantula, J.; Diez, J.J. Fusarium circinatum isolates from northern Spain are commonly infected by three distinct mitoviruses. Arch. Virol. 2015, 160, 2093-2098. [CrossRef]

20. Polashock, J.J.; Hillman, B.I. A small mitochondrial double-stranded (ds) RNA element associated with a hypovirulent strain of the chestnut blight fungus and ancestrally related to yeast cytoplasmic T and W dsRNAs. Proc. Natl. Acad. Sci. USA 1994, 91, 8680-8684. [CrossRef]

21. Tran, T.T.; Li, H.; Nguyen, D.Q.; Jones, M.G.K.; Wylie, S.J. Co-infection with three mycoviruses stimulates growth of a Monilinia fructicola isolate on nutrient medium, but does not induce hypervirulence in a natural host. Viruses 2019, 11, 89. [CrossRef]

22. Springer, J.C.; Davelos Baines, A.L.; Chansler, M.T.; Jarosz, A.M. Evaluating the long-term storage of Cryphonectria parasitica. Fungal Genet. Rep. 2013, 60, 11-15. [CrossRef]

23. Agrawal, N.; Dasaradhi, P.V.N.; Mohmmed, A.; Malhotra, P.; Bhatnagar, R.K.; Mukherjee, S.K. RNA Interference: Biology, Mechanism, and Applications. Microbiol. Mol. Biol. Rev. 2003, 67, 657-685. [CrossRef] [PubMed]

24. Hammond, T.M.; Andrewski, M.D.; Roossinck, M.J.; Keller, N.P. Aspergillus mycoviruses are targets and suppressors of RNA silencing. Eukaryot. Cell 2008, 7, 350-357. [CrossRef]

25. Chen, Y.; Gao, Q.; Huang, M.; Liu, Y.; Liu, Z.; Liu, X.; Ma, Z. Characterization of RNA silencing components in the plant pathogenic fungus Fusarium graminearum. Sci. Rep. 2015, 5, 1-13. [CrossRef] [PubMed]

26. Zhang, D.X.; Spiering, M.J.; Nuss, D.L. Characterizing the roles of Cryphonectria parasitica RNA-dependent RNA polymerase-like genes in antiviral defense, viral recombination and transposon transcript accumulation. PLoS ONE 2014, 9, e108653. [CrossRef]

27. Segers, G.C.; Zhang, X.; Deng, F.; Sun, Q.; Nuss, D.L. Evidence that RNA silencing functions as an antiviral defense mechanism in fungi. Proc. Natl. Acad. Sci. USA 2007, 104, 12902-12906. [CrossRef]

28. Sun, Q.; Choi, G.H.; Nuss, D.L. A single Argonaute gene is required for induction of RNA silencing antiviral defense and promotes viral RNA recombination. Proc. Natl. Acad. Sci. USA 2009, 106, 17927-17932. [CrossRef]

29. Himeno, M.; Maejima, K.; Komatsu, K.; Ozeki, J.; Hashimoto, M.; Kagiwada, S.; Yamaji, Y.; Namba, S. Significantly low level of small RNA accumulation derived from an encapsidated mycovirus with dsRNA genome. Virology 2010, 396, 69-75. [CrossRef] 
30. Donaire, L.; Ayllón, M.A. Deep sequencing of mycovirus-derived small RNAs from Botrytis species. Mol. Plant Pathol 2017, 18, 1127-1137. [CrossRef]

31. Vainio, E.J.; Jurvansuu, J.; Hyder, R.; Kashif, M.; Piri, T.; Tuomivirta, T.; Poimala, A.; Xu, P.; Mäkelä, S.; Nitisa, D.; et al Heterobasidion Partitivirus 13 Mediates Severe Growth Debilitation and Major Alterations in the Gene Expression of a Fungal Forest Pathogen. J. Virol. 2018, 92, 1-15. [CrossRef]

32. O'Connor, S.M.; Taylor, C.E.; Hughes, J.M. Emerging Infectious Diseases; Centers for Disease Control and Prevention (CDC): Atlanta, GA, USA, 2006; pp. 1051-1057.

33. Galeotti, C.; Bayry, J. Autoimmune and inflammatory diseases following COVID-19. Nat. Rev. Rheumatol. 2020, 16, 413-414. [CrossRef] [PubMed]

34. Muñoz-Adalia, E.J.; Flores-Pacheco, J.A.; Martínez-Álvarez, P.; Martín-García, J.; Fernández, M.; Diez, J.J. Effect of mycoviruses on the virulence of Fusarium circinatum and laccase activity. Physiol. Mol. Plant Pathol. 2016, 94, 8-15. [CrossRef]

35. Mortazavi, A.; Williams, B.A.; McCue, K.; Schaeffer, L.; Wold, B. Mapping and quantifying mammalian transcriptomes by RNA-Seq. Nat. Methods 2008, 5, 621-628. [CrossRef] [PubMed]

36. Hansen, K.D.; Irizarry, R.A.; Wu, Z. Removing technical variability in RNA-Seq data using conditional quantile normalization. Biostatistics 2012, 13, 204-216. [CrossRef]

37. Baggerly, K.A.; Deng, L.; Morris, J.S.; Aldaz, C.M. Differential expression in SAGE: Accounting for normal between-library variation. Bioinformatics 2003, 19, 1477-1483. [CrossRef]

38. Benjamini, Y.; Hochberg, Y. Controlling the False Discovery Rate: A Practical and Powerful Approach to Multiple. J. R. Stat. Soc. Ser. B 1995, 57, 289-300. [CrossRef]

39. Gaffar, F.Y.; Imani, J.; Karlovsky, P.; Koch, A.; Kogel, K.-H. Different Components of the RNA Interference Machinery Are Required for Conidiation, Ascosporogenesis, Virulence, Deoxynivalenol Production, and Fungal Inhibition by Exogenous Double-Stranded RNA in the Head Blight Pathogen Fusarium graminearum. Front. Microbiol. 2019, 10, 1662. [CrossRef]

40. Lee, H.-C.; Li, L.; Gu, W.; Xue, Z.; Crosthwaite, S.K.; Pertsemlidis, A.; Lewis, Z.A.; Freitag, M.; Selker, E.U.; Mello, C.C.; et al. Article Diverse Pathways Generate MicroRNA-like RNAs and Dicer-Independent Small Interfering RNAs in Fungi. Mol. Cell 2010, 38, 803-814. [CrossRef]

41. Conesa, A.; Götz, S.; García-Gómez, J.M.; Terol, J.; Talón, M.; Robles, M. Blast2GO: A universal tool for annotation, visualization and analysis in functional genomics research. Bioinformatics 2005, 21, 3674-3676. [CrossRef]

42. Jones, P.; Binns, D.; Chang, H.-Y.; Fraser, M.; Li, W.; Mcanulla, C.; Mcwilliam, H.; Maslen, J.; Mitchell, A.; Nuka, G.; et al. InterProScan 5: Genome-scale protein function classification. Bioinformatics 2014, 30, 1236-1240. [CrossRef]

43. Supek, F.; Bošnjak, M.; Škunca, N.; Šmuc, T. REVIGO Summarizes and Visualizes Long Lists of Gene Ontology Terms. PLoS ONE 2011, 6, e21800. [CrossRef] [PubMed]

44. Marioni, J.C.; Mason, C.E.; Mane, S.M.; Stephens, M.; Gilad, Y. RNA-Seq: An assessment of technical reproducibility and comparison with gene expression arrays. Genome Res. 2008, 18, 1509-1517. [CrossRef] [PubMed]

45. Wickramasinghe, S.; Rincon, G.; Islas-Trejo, A.; Medrano, J.F. Transcriptional profiling of bovine milk using RNA sequencing. BMC Genom. 2012, 13, 45. [CrossRef] [PubMed]

46. Vainio, E.J.; Jurvansuu, J.; Streng, J.; Rajamä, M.-L.; Hantula, J.; Valkonen, J.P.T. Diagnosis and discovery of fungal viruses using deep sequencing of small RNAs. J. Gen. Virol. 2015, 96, 714-725. [CrossRef]

47. Park, Y.; Chen, X.; Punja, Z.K. Molecular and Biological Characterization of a Mitovirus in Chalara elegans (Thielaviopsis basicola). Phytopathology 2006, 96, 468-479. [CrossRef]

48. Thekke Veetil, T.; Ho, T.; Moyer, C.; Whitaker, V.M.; Tzanetakis, I.E. Detection of strawberry necrotic shock virus using conventional and TaqMan®quantitative RT-PCR. J. Virol. Methods 2016, 235, 176-181. [CrossRef]

49. Zhang, S.; Vrient, A. Rapid detection of plant viruses and viroids. In Applied Plant Virology; Elsevier: Amsterdam, The Netherlands, 2020; pp. 101-109.

50. Barba, M.; Czosnek, H.; Hadidi, A. Historical Perspective, Development and Applications of Next-Generation Sequencing in Plant Virology. Viruses 2014, 6, 106-136. [CrossRef]

51. Jo, Y.; Lian, S.; Chu, H.; Cho, J.K.; Yoo, S.-H.; Choi, H.; Yoon, J.-Y.; Choi, S.-K.; Lee, B.C.; Cho, W.K. Peach RNA viromes in six different peach cultivars. Sci. Rep. 2018, 8, 1844. [CrossRef]

52. Jo, Y.; Choi, H.; Chu, H.; Cho, W.K. Identification of viruses from fungal transcriptomes. BioRxiv 2020, under review.

53. Lee, K.M.; Cho, W.K.; Yu, J.; Son, M.; Choi, H.; Min, K.; Lee, Y.W.; Kim, K.H. A comparison of transcriptional patterns and mycological phenotypes following infection of Fusarium graminearum by four mycoviruses. PLoS ONE 2014, 9, e100989. [CrossRef]

54. Cai, G.; Fry, W.E.; Hillman, B.I. PiRV-2 stimulates sporulation in Phytophthora infestans. Virus Res. 2019, 271, 197674. [CrossRef]

55. Wang, S.; Zhang, J.; Li, P.; Qiu, D.; Guo, L. Transcriptome-based discovery of Fusarium graminearum stress responses to FgHV1 infection. Int. J. Mol. Sci. 2016, 17, 1922. [CrossRef]

56. Kennedy, J.; Auclair, K.; Kendrew, S.G.; Park, C.; Vederas, J.C.; Hutchinson, C.R. Modulation of polyketide synthase activity by accessory proteins during lovastatin biosynthesis. Science 1999, 284, 1368-1372. [CrossRef] [PubMed]

57. Miedaner, T.; Gwiazdowska, D.; Waśkiewicz, A. Management of Fusarium species and their mycotoxins in cereal food and feed. Front. Microbiol. 2017, 8, 1543. [CrossRef] [PubMed]

58. Sorensen, J.L.; Auclair, K.; Kennedy, J.; Hutchinson, C.R.; Vederas, J.C. Transformations of cyclic nonaketides by Aspergillus terreus mutants blocked for lovastatin biosynthesis at the $\operatorname{lov} A$ and $\operatorname{lov} C$ genes. Org. Biomol. Chem. 2003, 1, 50-59. [CrossRef] 
59. Patkar, R.N.; Ramos-Pamplona, M.; Gupta, A.P.; Fan, Y.; Naqvi, N.I. Mitochondrial $\beta$-oxidation regulates organellar integrity and is necessary for conidial germination and invasive growth in Magnaporthe oryzae. Mol. Microbiol. 2012, 86, 1345-1363. [CrossRef] [PubMed]

60. Berepiki, A.; Lichius, A.; Read, N.D. Actin organization and dynamics in filamentous fungi. Nat. Rev. Microbiol. $2011,9,876-887$. [CrossRef]

61. Cheffings, T.H.; Burroughs, N.J.; Balasubramanian, M.K. Actomyosin ring formation and tension generation in eukaryotic cytokinesis. Curr. Biol. 2016, 26, 719-737. [CrossRef]

62. Kim, Y.; Park, S.Y.; Kim, D.; Choi, J.; Lee, Y.H.; Lee, J.H.; Choi, W. Genome-scale analysis of ABC transporter genes and characterization of the ABCC type transporter genes in Magnaporthe oryzae. Genomics 2013, 101, 354-361. [CrossRef]

63. Mohanta, T.K. Fungi contain genes associated with flavonoid biosynthesis pathway. J. Funct. Foods 2020, 68, 103910. [CrossRef]

64. Aguirre, J.; Ríos-Momberg, M.; Hewitt, D.; Hansberg, W. Reactive oxygen species and development in microbial eukaryotes. Trends Microbiol. 2005, 13, 111-118. [CrossRef] [PubMed]

65. Wang, Y.; Ji, D.; Chen, T.; Li, B.; Zhang, Z.; Qin, G.; Tian, S. Production, signaling, and scavenging mechanisms of reactive oxygen species in fruit-pathogen interactions. Int. J. Mol. Sci. 2019, 20, 2994. [CrossRef] [PubMed]

66. Gessler, N.N.; Aver'yanov, A.A.; Belozerskaya, T.A. Reactive oxygen species in regulation of fungal development. Biochemistry 2007, 72, 1091-1109. [CrossRef] [PubMed]

67. Allen, T.D.; Dawe, A.L.; Nuss, D.L. Use of cDNA microarrays to monitor transcriptional responses of the chestnut blight fungus Cryphonectria parasitica to infection by virulence-attenuating hypoviruses. Eukaryot. Cell 2003, 2, 1253-1265. [CrossRef] [PubMed]

68. Mcbride, R.C.; Boucher, N.; Park, D.S.; Turner, P.E.; Townsend, J.P.; Townsend, J. Yeast response to LA virus indicates coadapted global gene expression during mycoviral infection. FEMS Yeast Res. 2013, 13, 162-179. [CrossRef]

69. Chang, S.-S.; Zhang, Z.; Liu, Y. RNA Interference Pathways in Fungi: Mechanisms and Functions. Annu. Rev. Microbiol. 2012, 66, 305-323. [CrossRef]

70. Muñoz-Adalia, E.J.; Diez, J.J.; Fernandez, M.M.; Hantula, J.; Vainio, E.J. Characterization of small RNAs originating from mitoviruses infecting the conifer pathogen Fusarium circinatum. Arch. Virol. 2018, 163, 1009-1018. [CrossRef]

71. Shahi, S.; Eusebio-Cope, A.; Kondo, H.; Hillman, B.I.; Suzuki, N. Investigation of host range of and host defense against a mitochondrially replicating mitovirus. J. Virol. 2019, 93. [CrossRef] 